\title{
The Design of Educational Simulator for Noise and Filter Types in Image Processing
}

\author{
${ }^{* 1}$ Fahri Vatansever and ${ }^{1}$ Nedim Aktan Yalcin \\ ${ }^{1}$ Faculty of Engineering, Department of Electrical-Electronics Engineering, Uludag University, Turkey
}

\begin{abstract}
Noise and filtering noise are basic topics in the field of signal and image processing. In this study, an interactive simulator is designed for comprehension of related topics and to be reinforced theoretical knowledge with practical applications by students which have educated in these fields. Knowledge about noise and filtering in the field of image processing is easily, quickly and effectively gained to students with the simulator which includes many noise (Gaussian, Gamma, Rayleigh etc.) and filter (mean, median, min, max etc.) types, presents theoretical topics (explanations, mathematical statements, examples, etc.) about them visually, allows realizing of interactive applications (parameter and type changes etc.) and creates singular and comparative performance analysis.
\end{abstract}

Key words: Image processing, noise, filter, simulator.

\section{Introduction}

Image processing is one of basic topics in electronics and computer world. Especially, developments in computer sciences lead increase of digital image processing applications. In order to obtain maximum performance for this type of applications, it is necessary to be used undistorted images. However, obtaining, processing coding and transmission of images cause noise on them. Noise cleaning without losing information is crucial operation and in this area, there are many methods, algorithms, transforms, filters etc. The basic purpose of this topic is choosing the best noise cleaning methods according to different noise types for obtaining the highest performance [1-10].

Supporting theoretical knowledge with applications simplifies understanding and comprehension of related topics. Therefore, utilization of multimedia instruments and simulators increases success rate in theoretical lessons. There are many studies for teaching image processing topics in this area [11-14].

In realized study, educational simulator is designed, for noise types in digital images and cleaning them. There are many applications and educational explanations in interactive simulator which includes many noises and filter types and has user-friendly interface. Thus, users which want to comprehend various topics in this area can easily and quickly observe effects of different filters and parameters thanks to its visual and interactive applications.

\section{The Noise Types/Models}

Noises are undesirable data in digital images and harm image quality. Some basic noise types and its properties in digital images are given in Table 1[1]. Except those given models in Table 1,

*Corresponding author: Address: Faculty of Engineering, Department of Electrical-Electronics Engineering, Uludag University, 16059, Bursa, TURKEY. E-mail address: fahriv@uludag.edu.tr, Phone: +902242940905 
there are many different noise types such as speckle, Brownian (fractal), Poisson-Gauss, periodic, structure borne, quantization, white etc. noise types.

Table 1. The some noise types

\begin{tabular}{|c|c|c|c|}
\hline Noise & Probability density function (PDF) & Average value & Variance \\
\hline Gaussian & $p(z)=\frac{1}{\sqrt{2 \pi} \sigma} e^{-\frac{(z-\mu)^{2}}{2 \sigma^{2}}}$ & $\mu$ & $\sigma^{2}$ \\
\hline Rayleigh & $p(z)=\left\{\begin{array}{cc}\frac{2}{b}(z-a) e^{-\frac{(z-a)^{2}}{b}}, & z \geq a \\
0 & , \quad z<0\end{array}\right.$ & $\mu=a+\sqrt{\frac{\pi b}{4}}$ & $\sigma^{2}=\frac{b(4-\pi)}{4}$ \\
\hline $\begin{array}{l}\text { Gamma } \\
\text { (Erlang) }\end{array}$ & $p(z)=\left\{\begin{array}{cc}\frac{a^{b} z^{b-1}}{(b-1) !} e^{-a z} & , \quad z \geq 0 \\
0 & , \quad z<0\end{array}\right.$ & $\mu=\frac{b}{a}$ & $\sigma^{2}=\frac{b}{a^{2}}$ \\
\hline Exponential & $p(z)=\left\{\begin{array}{cc}a e^{-a z} & , \quad z \geq 0 \\
0 & , \quad z<0\end{array}\right.$ & $\mu=\frac{1}{a}$ & $\sigma^{2}=\frac{1}{a^{2}}$ \\
\hline Uniform & $p(z)=\left\{\begin{array}{ccc}\frac{1}{b-a} & , & a \leq z \leq b \\
0, & \text { diğer }\end{array}\right.$ & $\mu=\frac{a+b}{2}$ & $\sigma^{2}=\frac{(b-a)^{2}}{12}$ \\
\hline $\begin{array}{l}\text { Impulse } \\
\text { (salt-and- } \\
\text { pepper) }\end{array}$ & $p(z)=\left\{\begin{array}{cc}P_{a} & , \quad z=a \\
P_{b}, & z=b \\
0, & \text { di } \text { ger }\end{array}\right.$ & & \\
\hline
\end{tabular}

\section{The Filter Types}

Many filter types are used in noise cleaning processes whose basic purpose is erasing noise in images and provide the highest quality images. Besides class of linear and non-linear de-noising filter types, there are another class which includes mean, order-statistics and adaptive filter types. Some filter types are given in Table 2[1].

Table 2. The some filter types

\begin{tabular}{|c|c|c|}
\hline & Filters & Expression \\
\hline \multirow{4}{*}{ 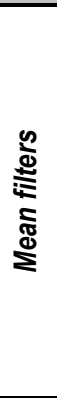 } & Arithmetic mean & $\hat{f}(x, y)=\frac{1}{m n} \sum_{r, s \in A_{x y}} g(r, s)$ \\
\hline & Geometric mean & $\hat{f}(x, y)=\sqrt[m n]{\prod_{r, s \in A_{x y}} g(r, s)}$ \\
\hline & Harmonic mean & $\hat{f}(x, y)=\frac{m n}{\sum_{r, s \in A_{x y}} \frac{1}{g(r, s)}}$ \\
\hline & Contraharmonic mean & $\hat{f}(x, y)=\frac{\sum_{r, s \in A_{x y}} g(r, s)^{q+1}}{\sum_{r, s \in A_{x y}} g(r, s)^{q}}$ \\
\hline \multirow{5}{*}{ 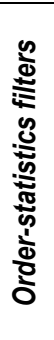 } & Median & $\hat{f}(x, y)=\operatorname{median}_{r, s \in A_{x y}}\{g(r, s)\}$ \\
\hline & $\operatorname{Max}$ & $\hat{f}(x, y)=\max _{r, s \in A_{x y}}\{g(r, s)\}$ \\
\hline & Min & $\hat{f}(x, y)=\min _{r, s \in A_{x y}}\{g(r, s)\}$ \\
\hline & Midpoint & $\hat{f}(x, y)=\frac{1}{2}\left\{\max _{r, s \in A_{x y}}\{g(r, s)\}+\min _{r, s \in A_{x y}}\{g(r, s)\}\right\}$ \\
\hline & Alpha-trimmed mean & $\hat{f}(x, y)=\frac{1}{m n-d} \sum_{r, s \in A_{x y}} g_{k}(r, s)$ \\
\hline \multicolumn{3}{|c|}{$\begin{array}{l}A_{x y}: \text { Set of coordinates in a rectangular sub-image window of size } m \times n \text {, centered at point }(x, y) \\
g(x, y) \text { : The corrupted image in the area defined by } A_{x y} \\
f(x, y) \text { : The value of restored image at point }(x, y)\end{array}$} \\
\hline
\end{tabular}




\section{The Designed Simulator and Applications}

Educational and interactive simulator which includes noise types and de-noising filters in image processing is designed with MATLAB[15]. Designed simulator allows adding different types of noise to uploaded or captured images and regeneration of these images with selected filters. Besides, it contains detailed explanations about noise and filter types. Additionally, it provides realization of comparative simulations for de-noising process.

Menu items of educational simulator can be seen in Table 3. Additionally, properties of toolbar which are located near main screen is explained in Figure 1.

Table 3. The some main menu contents of designed simulator

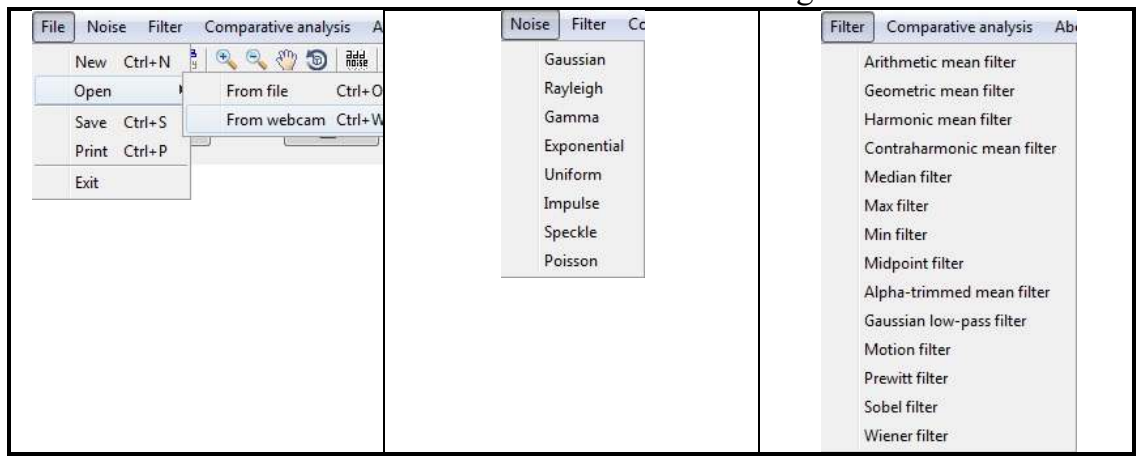

\begin{tabular}{|c|c|c|c|c|c|c|c|c|c|c|c|}
\hline 45 & +3 & $1=1$ & tas & $\begin{array}{l}\text { PGB } \\
\text { Gray }\end{array}$ & +4 & -4 & \& & t要 & 吊品品 & + & 1 \\
\hline$\frac{3}{2}$ & ్ㅡㅇ & $\underset{\infty}{\stackrel{\infty}{\infty}}$ & 를 & $\begin{array}{l}\text { tั. } \\
\stackrel{0}{0} \\
\text { Oे }\end{array}$ & 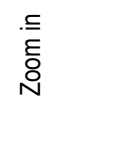 & $\begin{array}{l}\text { క̄ } \\
\text { E } \\
\text { ㅇ }\end{array}$ & ๙ & 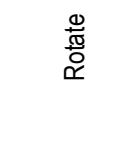 & $\begin{array}{l}\frac{D}{0} \\
\frac{.0}{0} \\
\frac{0}{0} \\
\frac{0}{2}\end{array}$ & 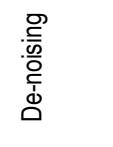 & 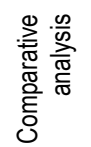 \\
\hline
\end{tabular}

Figure 1. The toolbar of designed simulator

In Figure 2, explanation about a specific noise type can be seen. In this module, PDF of noise can be plotted according to selected parameters. Furthermore noise can be added to uploaded or captured images, histograms and spatial domain values of original and distorted images can be plotted and mean and standard deviation values can be printed.

In Figure 3, adding noise and de-noising steps are presented. Firstly, image was uploaded from file, or captured from camera (Fig. 3a). Secondly, noise type was selected and desired parameters were set (Fig. 3b). Thirdly, filter type and window size were specified (Fig. 3c). Thus, uploaded or captured image was distorted by noise, and de-noised with selected filters. Besides, meansquared error (MSE) and peak signal-to-noise ratio (PSNR) were calculated and showed in order to observing performance of process (Fig. 3d). 


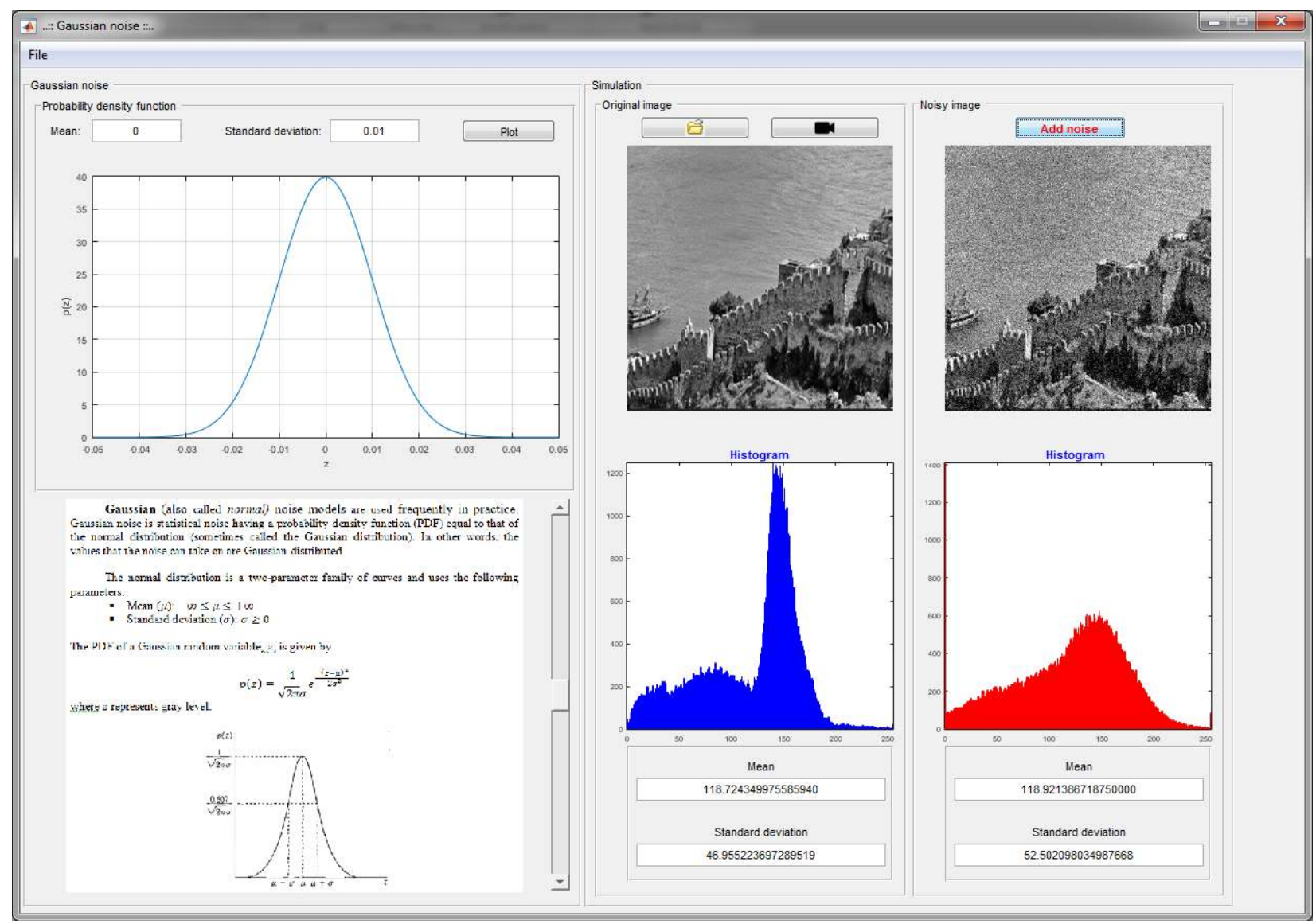

Figure 2. The sample screenshot for noise types

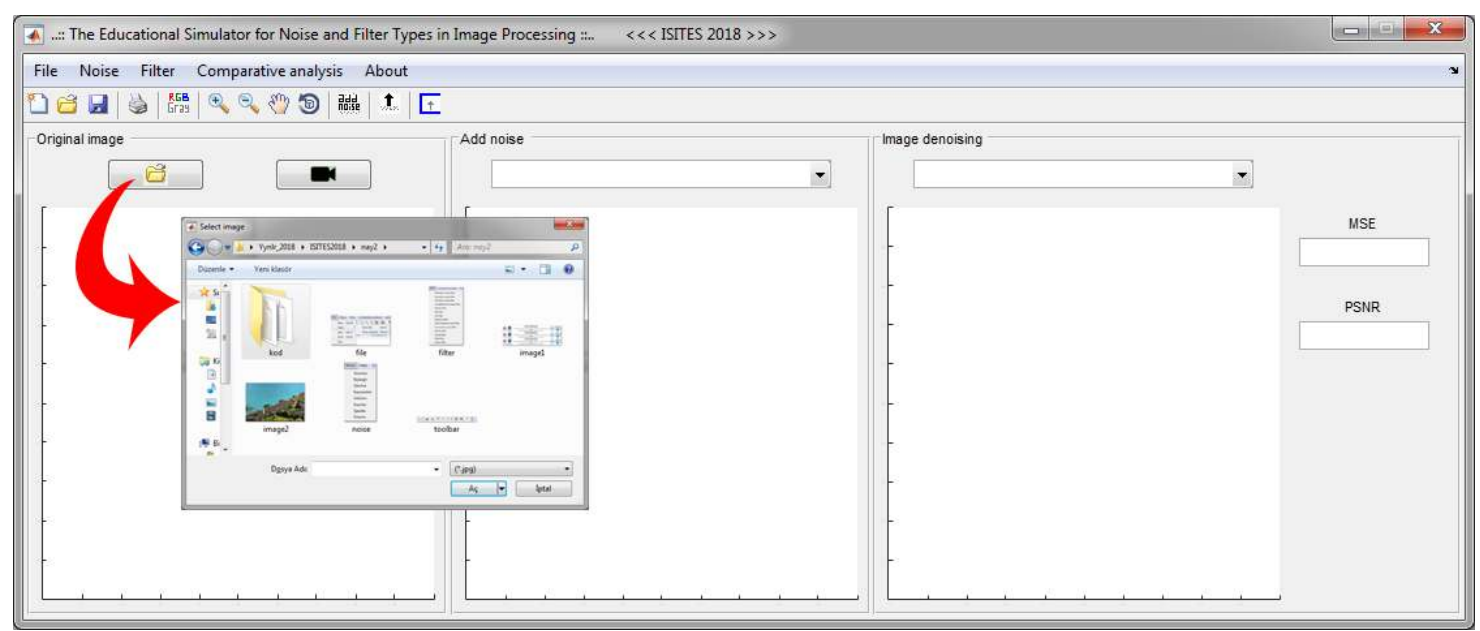

(a) 


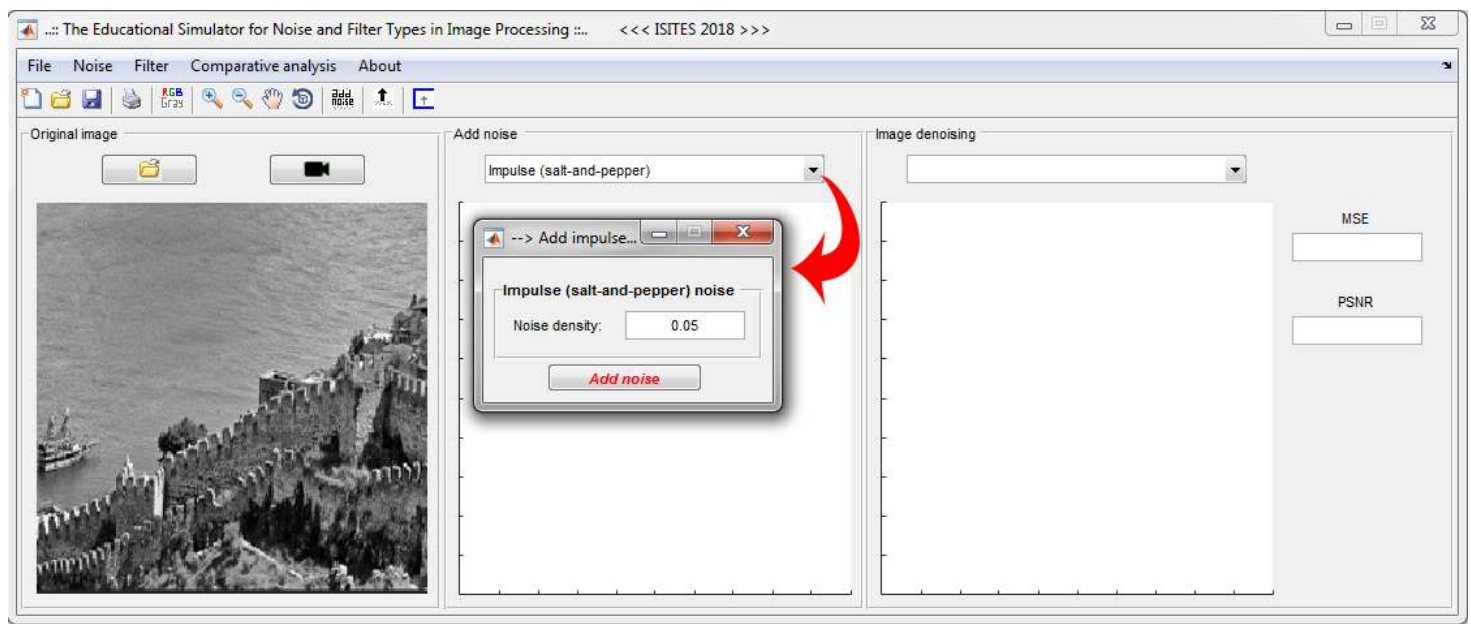

(b)

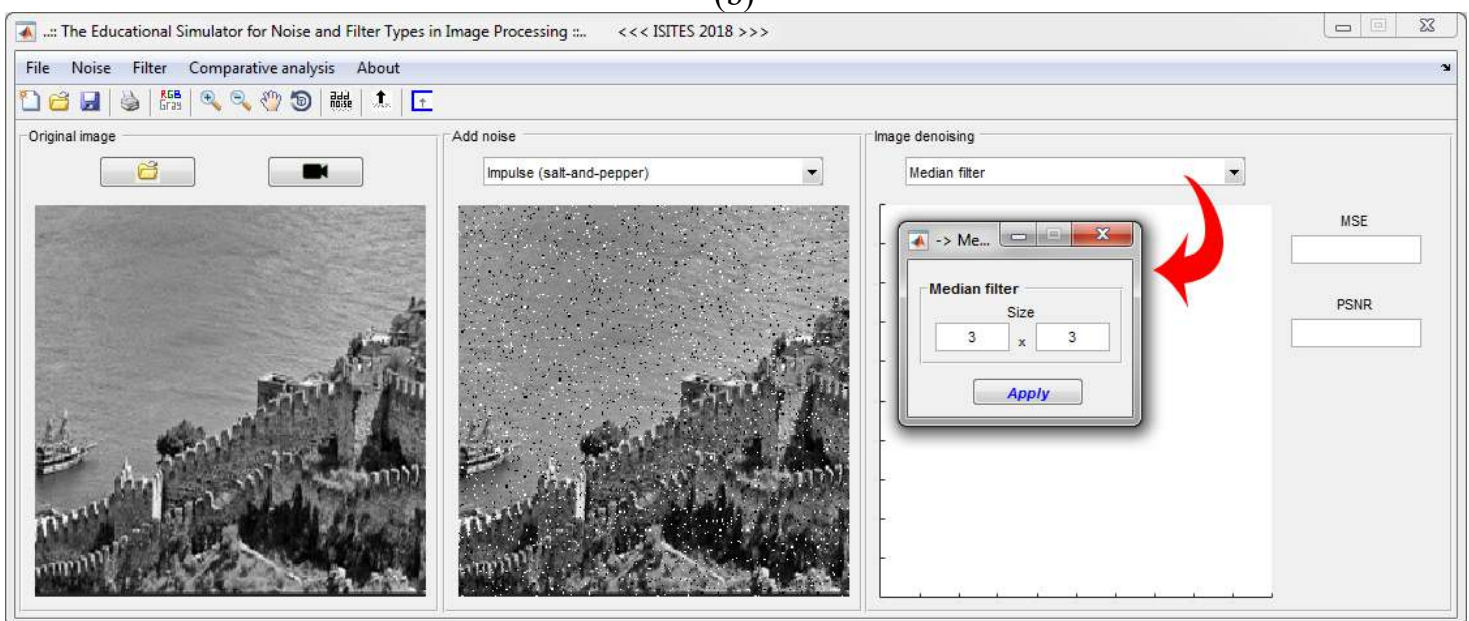

(c)

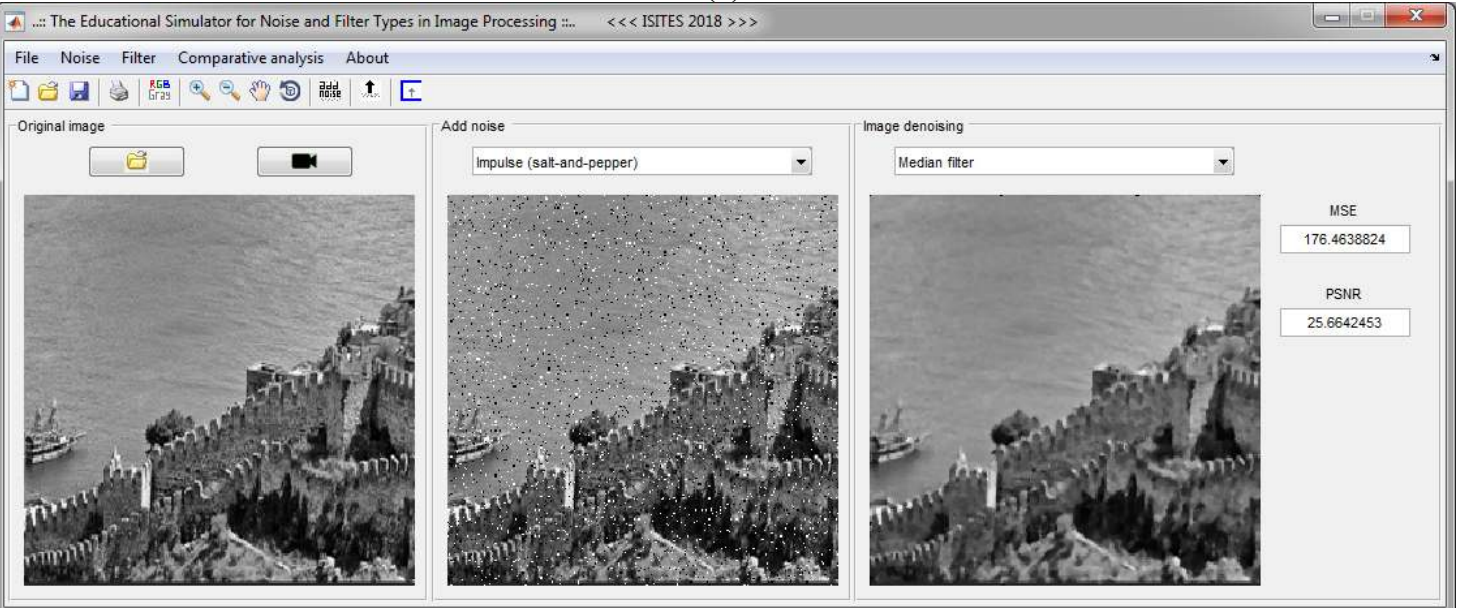

(d)

Figure 3. The sample screenshots of de-noising process steps 


\section{Conclusions}

In realized study, educational simulator is designed for noise and filter types. With simulator which includes interactive modules and explanations about simulations, specified noise can be added to images and this noise can be erased with appropriate filters. Therefore, effects of noise, filters and filter parameters can be seen clearly and comparative simulations ensure more comprehensive learning.

\section{References}

[1] Gonzales RC, Woods RE. Digital image processing. 2nd ed. The elements of style. 3rd ed. New Jersey: Prentice HAll; 2002.

[2] Bovik A (Ed.). Handbook of image\&video processing. Canada: Academic Press; 2000.

[3] Boyat AK, Joshi BK. A review paper: Noise models in digital image processing. Signal\&Image Processing: An International Journal 2015; 6 (2): 63-75.

[4] Kaur S. Noise types and various removal techniques. International Journal of Advanced Research in Electronics and Communication Engineering 2015; 4(2): 226-230.

[5] Patidar P, Gupta M, Srivastava S, Nagawat AK. Image de-noising by various filters for different noise. International Journal of Computer Applications 2010; 9(4): 45-50.

[6] Kaur G, Kumar R, Kainth K. A review paper on different noise types and digital image processing. International Journal of Advanced Research in Computer Science and Software Engineering 2016; 6(6): 562-565.

[7] Verma R, Ali J. A comparative study of various types of image noise and efficient noise removal techniques. International Journal of Advanced Research in Computer Science and Software Engineering 2013; 3(10): 617-622.

[8] Suresh SS. A survey on types of noise model, noise and denoising technique in digital image processing. International Journal of Innovative Research in Computer and Communication Engineering 2017; 5(2): 50-56.

[9] Sontakke MD, Kulkarni MS. Different types of noises in images and noise removing technique. International Journal of Advanced Technology in Engineering and Science 2015; 3 (1): 102-115.

[10] Farooque MA, Rohankar JS. Survey on various noises and techniques for denoising the color image. International Journal of Application or Innovation in Engineering \& Management 2013; 2(11): 217-221.

[11] Ramirez JM, Garcia-Aguilar RA, Báez-López D. ProDIM: Educational software for digital image processing. Computer Applications in Engineering Education 1995; 3(3): 165-172.

[12] Yapp CHW, See AKB. Teaching image processing: A two-step process. Computer Applications in Engineering Education 2008; 16(3): 211-222.

[13] Ayala M, Adjouadi M, Cabrerizo M, Barreto A. A Windows-based interface for teaching image processing. Computer Applications in Engineering Education 2010; 18(2): 213-224.

[14] Ramirez JM, Garcia-Aguilar RA, Báez-López D. ProDIM: Educational software for digital image processing. Computer Applications in Engineering Education 1995; 3(3): 165-172.

[15] MATLAB, The MathWorks Inc. Co. 